\title{
O uso de Data Mart para apoio à tomada de decisão na Gestão de Pessoas em uma Instituição Federal de Ensino
}

\author{
Edivaldo da Silva Souza ${ }^{1}$, Luiz Antônio Abrantes², Jugurta Lisboa-Filho' \\ ${ }^{1}$ Departamento de Informática, ${ }^{2}$ Departamento de Administração \\ Universidade Federal de Viçosa \\ Viçosa, Minas Gerais, Brasil \\ edivaldo.souza@ufv.br, abrantes@ufv.br,jugurta@ufv.br
}

\begin{abstract}
Business Intelligence (BI) consists of a multidimensional database, oriented by subject, non-volatile, historical, decision-making and variable in relation to time. When applying the use of Data Mart to a Federal Education Institution in the people management sector, this research worked with the data to develop indicators for decision making. Issues such as lack of synergy between the existing databases, quality of the data provided and the impossibility of issuing timely management reports, were addressed in this study. It was concluded that the application and implementation of BI through Data Mart can generate accurate data, solve problems and provide performance indicators.
\end{abstract}

Resumo. Business Intelligence (BI) é composto por um banco de dados multidimensional, orientado por assunto, não volátil, histórico, decisório e variável em relação ao tempo. Ao aplicar o uso do Data Mart para uma Instituição Federal de Ensino no setor de gestão de pessoas, essa pesquisa trabalhou com os dados para desenvolver indicadores para a tomada de decisão. Questões como falta de sinergia entre as bases de dados existentes, qualidade dos dados fornecidos e impossibilidade da emissão de relatórios gerenciais em tempo hábil, foram tratados nesse estudo. Concluiu-se que a aplicação e implantação de BI através de Data Mart pode gerar dados precisos, solucionar os problemas e fornecer indicadores de desempenho.

\section{Introdução}

As Instituições Federais de Ensino (IFEs) passaram por grandes mudanças e expansões nas últimas duas décadas. O marco regulatório se deu através do Decreto $n^{\circ}$ 6.096/2007 (BRASIL, 2007) que criou o Programa de Apoio a Planos de Reestruturação e Expansão das Universidades Federais - REUNI, que teve como objetivo ampliar o acesso e a permanência na educação superior. As ações do programa contemplam o aumento de vagas nos cursos de graduação, a ampliação da oferta de cursos noturnos, a promoção de inovações pedagógicas e o combate à evasão, entre outras metas que têm o propósito de diminuir as desigualdades sociais no país.

Consequentemente, as mudanças provocadas pelo REUNI nas IFEs criaram novos desafios para o processo de gestão, considerando que no mesmo momento que se apontava para expansão restringia-se os recursos financeiros e humanos. A criação do Quadro de Referência dos Servidores Técnico-Administrativos - QRSTA, pelo Decreto 
$\mathrm{n}^{\circ}$ 7.232/2010 (BRASIL, 2010), ao mesmo tempo que possibilitou a reposição automática de vagas por meio de Concurso Público impediu a reposição para os cargos de níveis "A" e "B" e para cargos de outros níveis que estariam extintos ou em extinção.

No caso dos servidores pertencentes a carreira de Magistério Superior, o Decreto $\mathrm{n}^{\mathrm{o}}$ 7.485/2011 (BRASIL, 2011) dispôs sobre a constituição de Banco de Professor Equivalente - BPEq concedendo autonomia às IFEs para gerenciar o seu quadro de pessoal docente. Entretanto, não se considerou todos os cargos vagos na apuração do quantitativo de vagas autorizadas. $\mathrm{O}$ mesmo procedimento foi adotado para a apuração dos cargos de técnico administrativo. Foram apurados, em 30 de junho de 2010, a partir da extração do Sistema Integrado de Administração de Recursos Humanos - SIAPE somente o quantitativo dos cargos ocupados naquela data.

Além disso, a reforma administrativa, que teve início com a reforma da previdência, ocasionou um crescimento das aposentadorias reduzindo parte da força de trabalho. Contabiliza-se também grande número de servidores, distribuídos por diversos setores, que já possuem tempo para solicitar o benefício.

Estes fatos acabaram imputando restrições na gestão financeira, operacional e principalmente na gestão de pessoal considerando a manutenção e até mesmo crescimento das demandas existentes e a dificuldade de reposição imediata de cargos que ainda tem seu provimento garantido por lei.

Diante deste cenário, as instituições federais de ensino, em especial o setor de gestão de pessoas da Universidade Federal de Viçosa (UFV), precisam implementar e aplicar tecnologias de gestão de informação com potencial para gerar dados confiáveis para auxiliar no processo decisório. A qualidade e a precisão da informação relacionada ao perfil socioeconômico dos servidores, a composição e localização espacial da estrutura física e operacional dos diversos setores existentes na Universidade torna-se uma importante fonte para o planejamento e tomada de decisões. Os dados podem ser obtidos por meio de consultas ao Sistema Integrado de Administração de Pessoal (SIAPE), do Governo Federal e ao Sistema de Dados de Recursos Humanos (SISREC), desenvolvido pela Instituição, ambos alimentados manualmente e não explorados de forma analítica.

Um dos grandes problemas encontrados nestas bases é a falta de sinergia entre elas, na qualidade dos dados fornecidos e na impossibilidade da emissão de relatórios gerenciais em tempo hábil, acrescidos de temporalidade na ocorrência de diversos eventos relacionados à gestão de pessoas.

Takashina e Flores (1996) afirmam que os indicadores são uma ferramenta essencial que auxiliam no planejamento e no controle de processos. Nesta ótica os indicadores de desempenho e o conceito de Business Intelligence (BI), com suas ferramentas inteligentes de negócios, têm sido considerados uma estratégica positiva dentro de uma organização, que geram informações precisas e sólidas, sendo, assim, essenciais para um processo decisório mais eficiente.

Para corroborar com este artigo podemos destacar Medina et al. (2018), que descrevem o uso de ferramentas de BI em uma universidade chilena para o desenvolvimento de um Data Mart com o intuito de obter indicadores de produtividade que auxiliem os gestores na tomada de decisão estratégica. O projeto envolveu quatro etapas que são: Análise; Projeto Conceitual; Processo ETL + V; e o Processo analítico. Como resultado obteve-se o desenvolvimento de uma plataforma de inteligência de 
negócios que auxiliou os gestores na tomada de decisão e permitiu a geração de novos indicadores.

O objetivo desse artigo foi apresentar as etapas do projeto e implementação de um Data Mart, utilizando ferramentas de BI de código aberto, para integração de base de dados, a fim de imprimir agilidade e fornecer subsídios para a tomada de decisão relacionadas ao controle e desenvolvimento de pessoas em uma IFEs.

Além desta Introdução, este artigo está estruturado em seções. A seção 2 descreve os principais conceitos de $\mathrm{BI}$ utilizados no projeto. O desenvolvimento do Data Mart é descrito na seção 3. A seção 4 apresenta os resultados da pesquisa e, finalmente, a seção 5 apresenta as considerações finais.

\section{Business Intelligence}

Business Intelligence (ou Inteligência de Negócios - BI) é um conjunto de métodos e tecnologias amplo, coeso e integrado de ferramentas e processos utilizados para dar suporte à tomada de decisão. Atualmente qualquer organização necessita de informações precisas que podem ser obtidas a partir de BI.

Neste contexto Primak (2008, p. 5) afirma que BI é "um processo inteligente de coleta, organização, análise, compartilhamento e monitoração de dados contidos em Data Warehouse (DW) e/ou Data Mart (DM), gerando informações para suporte à tomada de decisões no ambiente de negócios".

Sua estrutura, que compreende os Dados operacionais, Ferramentas ETL, Data Warehouse e On-line Analytical Processing, está descrita nas seções seguintes.

\subsection{Dados Operacionais}

A eficiência de BI depende da qualidade dos dados coletados no nível operacional. Essa fase reúne diversas fontes de dados distintas, como sistemas OLTP, sistema legados, tabelas com extensão xls, arquivos txt, XML e outros, para o processo de extração, transformação e carregamento dos dados no DW.

\subsection{Ferramentas ETL}

O processo de Extração, Transformação e Carga (ETL) é uma das primeiras fases do processo de $\mathrm{BI}$, onde várias fontes de dados como banco de dados transacionais, planilhas eletrônicas, arquivos de texto, XML e outras podem passar pelos processos de extração, limpeza, processamento e serem transformados e logo depois serem inseridos no DW e nos seus DM.

Kimball (1998) afirma que a extração de dados do processo ETL consome mais ou menos sessenta por cento do tempo de desenvolvimento de um DW, baseando em busca de informações mais importantes em várias fontes de dados.

\subsection{Data Warehouse - DW}

Data Warehouse é uma tecnologia utilizada por empresas privadas e entidades governamentais para ter sucesso na gestão e análise de dados. Neste contexto, DW é conhecido como armazém de dados históricos, tem o seu objetivo em disponibilizar informações precisas e eficientes para auxiliar nas tomadas de decisão nas organizações (Machado, 2007). 
Inmon (1997), define o termo como "um conjunto de dados baseado em assuntos, integrado, não volátil e variável em relação ao tempo, de apoio às decisões gerenciais". O DW é uma base de dados multidimensional com visão analítica, podendo integrar dados de diferentes fontes, unificando-os e facilitando a análise das informações. Os bancos de dados multidimensionais são divididos em tabelas "fatos" e "dimensões".

\subsection{On-line Analytical Processing - OLAP}

On-line Analytical Processing (OLAP) é um conjunto de ferramentas que permite a análise dos dados de um DW de forma dimensional (Machado, 2007). Os administradores das organizações têm acesso aos dados de forma rápida e eficaz, auxiliando nas tomadas de decisões.

Segundo Rob e Coronel (2010), OLAP apresenta quatro principais características: utiliza técnicas de análise de dados multidimensionais; estabelece suporte avançado a banco de dados; fornece interface fácil ao usuário final; e dá suporte a arquitetura cliente/servidor.

\section{Desenvolvimento do Data Mart}

Para o desenvolvimento do Data Mart foi utilizada a base de dados da Pró-Reitoria de Gestão de Pessoas (PGP) da Universidade Federal de Viçosa (UFV), de onde foram extraídos dados quantitativos dos servidores ativos no período de novembro de 2020 a fevereiro de 2021.

A primeira etapa da pesquisa foi realizar o processo de modelagem conceitual do banco de dados, onde foi analisada a base de dados RH, além de reuniões com os gestores que resultaram nos seguintes indicadores dos servidores: Por faixa etária e tempo de serviço; e por lotação de acordo com a situação dos cargos. Após esse levantamento, obteve-se os dados necessários para o desenvolvimento do diagrama UML. Em seguida, foi desenvolvida a modelagem utilizando o modelo floco de neve do processo BI, conforme proposto por Machado (2007).

A ferramenta escolhida para modelagem UML foi a StartUML 5.0, obtida no sítio http://www.dpi.ufv.br/projetos/geoprofile/download.html, sistema open source, que apresenta suporte para a modelagem de banco de dados espaciais. Em seguida depois de instalada, a ferramenta foi configurada para aceitar a modelagem de banco de dados geográficos utilizando o Perfil UML GeoProfile (Lisboa-Filho et al., 2010).

Para a execução do BI foram utilizadas as seguintes ferramentas: Processo ETL Pentaho Data Integration (PDI Kettle); cubo OLAP - Schema Workbench Mondrian; e dashboard - CDE Dashboard. Todas as ferramentas são open source, Versão 8.3 e estão disponíveis no sítio https://sourceforge.net/projects/pentaho da suíte Pentaho. Para o desenvolvimento do banco de dados multidimensional foi utilizado o SGDB PostgreSQL (Versão 12.3), com a extensão PostGIS para dados geográficos.

$\mathrm{O}$ resultado da modelagem multidimensional é ilustrado na Figura 1 com o diagrama UML+GeoProfile utilizando a ferramenta StarUML, apresentando uma tabela fato, conectada a dez tabelas dimensões (prefixo DIM) DIM_Funcionário, Faixa Etária, Status Tempo, Sexo, Tempo, Cargo, Cargo Status, Lotação (dimensão geográfica com representação espacial pontual), Campus (dimensão geográfica com representação espacial pontual) e Servidor. Apresenta ainda uma tabela Histórico Averbação que está 
ligada apenas na tabela Funcionário, que armazena o histórico de averbação de tempo de serviço dos servidores.

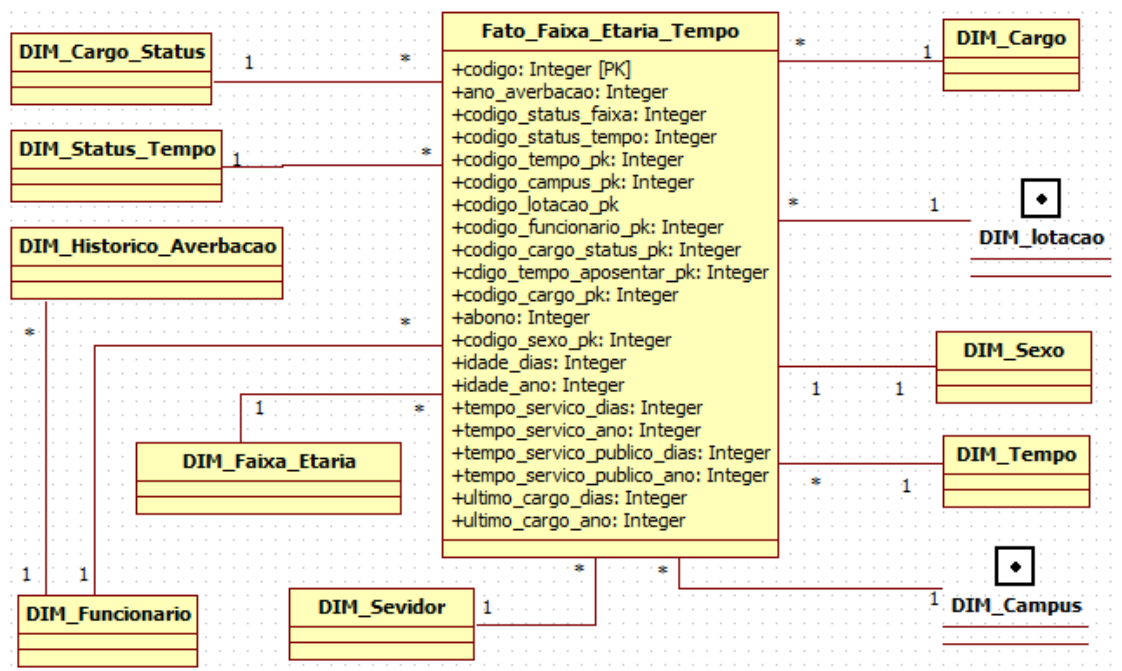

Figura 1 - Modelagem Multidimensional

Em seguida, foi realizado o processo ETL para fazer a conexão com banco de dados de Recursos Humanos (SISREC), concomitante a criação de planilhas eletrônicas para executar a carga das tabelas temporárias no DW. Para efetuar a carga na tabela fato Fato_Faixa_Etaria_Tempo foram criadas as tabelas temporárias dimensões (prefixo STG): STG_Funcionario, Cargo, Cargo Status, Lotação, Servidor, Histórico Averbação, Campus, Sexo, Status Faixa Etária, Status Tempo e Tempo. As tabelas Funcionário, Lotação e Histórico Averbação conectam-se à base de dados do RH. As demais tabelas conectam-se a arquivos CSV. A carga das tabelas temporárias é realizada em horário de menor demanda de acesso à base de dados. Esse processo é realizado pelo agendador de um job da ferramenta Pentaho, programado para executar essa rotina, no primeiro dia de cada mês. Com isso, a carga das tabelas de dimensões DIM no DW pode ser criada a qualquer momento, sem afetar o desempenho do banco de dados operacional.

Em seguida foi realizado um processo ETL para a criação e carga de cada tabela dimensão (prefixo DIM): DIM_Funcionario, Cargo, Cargo_Status, Lotacao, Servidor, Campus, Status_Faixa_Etaria, Sexo, Servidor, Tempo. A Tabela Averbação Histórico também foi criada e realizada a sua carga. Na Figura 1 existem 2 tabelas com propriedade de localização espacial: Lotacao e Campus. Para obter os dados geográficos dessas tabelas, utilizou-se a plataforma OpenStreetMap (OSM), disponível no sítio https://www.openstreetmap.org, onde previamente foi feito o mapeamento das lotações dos diversos órgãos e setores dos Campi Florestal, Rio Paranaíba e Viçosa, com as siglas de cada setor para efetuar as consultas pela API Nomination dos dados do OSM. A Figura 2 ilustra esse processo.

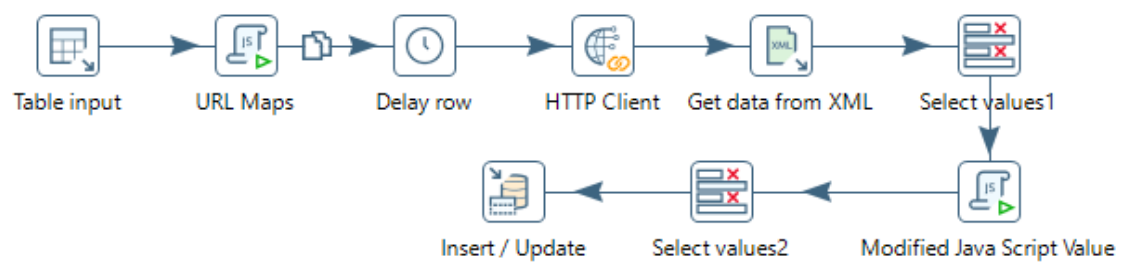

Figura 2: Geocodificação Nomination com dados OpenStreetMap Lotações 
O Step Table_Input (Figura 2) conecta-se à base de dados da tabela DIM_Lotacao, de onde os dados são extraídos. No Step URL Maps, é montado o caminho com o endereço da API do Nomination, conforme especificado no Código 1, onde a variável "url_final" corresponde à composição de um link da API Nomination com dados do OSM. Destaca-se que esse link é composto pelo endereço do sítio Nomination, sigla lotação, cidade, uf, format - que define o tipo de resposta no formato XML, polygon igual a "zero" indicando o não retorno ao polígono, addressdetails - igual a "zero" não retorna detalhes sobre o endereço, e limit - igual a "1", traz apenas o resultado mais próximo da pesquisa.

var url_final = 'https://nominatim. openstreetmap.org/search?q=' +encodeJRI(sigla)

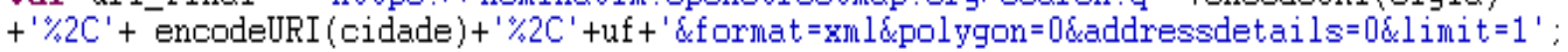

\section{Código 1. Geolocalização Nominatim com dados do OpenStreetMap}

O Step Delay row (Figura 2) é configurado de acordo com as restrições da API, que estabelece uma requisição por segundo. Em seguida o Step HTTP Client utiliza a configuração da URL final, solicita a requisição na API, codifica e configura a resposta de saída. O Step GET data from XML recebe a resposta da HTTP Client em formato XML, configura o caminho da requisição na Aba Content, opção Loop XPath, assim apresentado “/searchresults/place". Na aba Fields o campo XPath é configurado com os campos lat e lon selecionados da resposta da requisição. O tratamento dos dados é efetuado nos Step Select value1, Modified Java Scrit Value e Select value2. No Step Insert / Update é realizada a carga das coordenadas geográficas na tabela DIM_Lotação no DW. O mesmo procedimento foi utilizado para a tabela DIM_Campus para obter as coordenadas geográficas. Para verificar se ficou alguma lotação sem as informações de coordenadas geográficas, foi realizado uma rotina no processo ELT para verificar, caso tenha é disparado um email para o administrador.

Em seguida, foi realizada a carga no DW alimentando a tabela fato com valores relacionados com as tabelas dimensões. A Figura 3 ilustra o processo realizado para a carga na Tabela Fato.

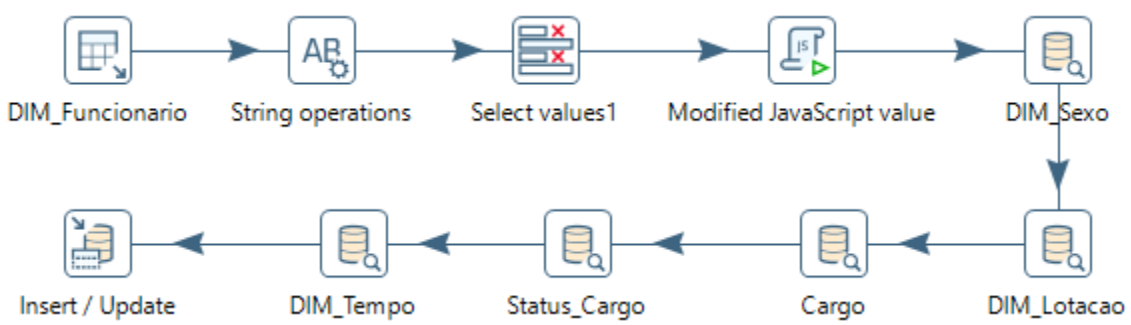

Figura 3 - Processo ETL Tabela "Fato_Faixa_Etaria_Tempo"

No Step DIM_Funcionario (Figura 3) é feita a conexão com a tabela DIM_Funcionário que busca apenas os servidores, classifica por classe e efetua o cálculo da faixa etária e do tempo de serviço, com averbação. Os Steps String operation, Select values 1 e Modified JavaScript value efetuam o tratamento dos dados. No Step DIM_Sexo, seleciona-se o código da tabela Dim_Sexo. Nos Steps DIM_Lotação, Cargo, Status_Cargo e DIM Tempo foram realizados os mesmos procedimentos, conectando-os nas tabelas de dimensões DIM_Lotacao, DIM_Cargo, DIM_Status_Cargo e DIM_Tempo, respectivamente. Finalmente, no Step Insert update, realizou-se a carga na Tabela Fato, resultando no DW. 
Após a carga dos dados na Tabela Fato utilizou-se a ferramenta Schema Workbench Mondrian para criar o cubo OLAP (Figura 4). A estrutura do cubo foi definida com base da modelagem multidimensional da Figura 1. Na ferramenta foi criado um Schema, no qual foi adicionado um cubo denominado "Banco_RH_Pesquisa" e dentro dele a tabela Fato "fato_faixa_etaria_servico".

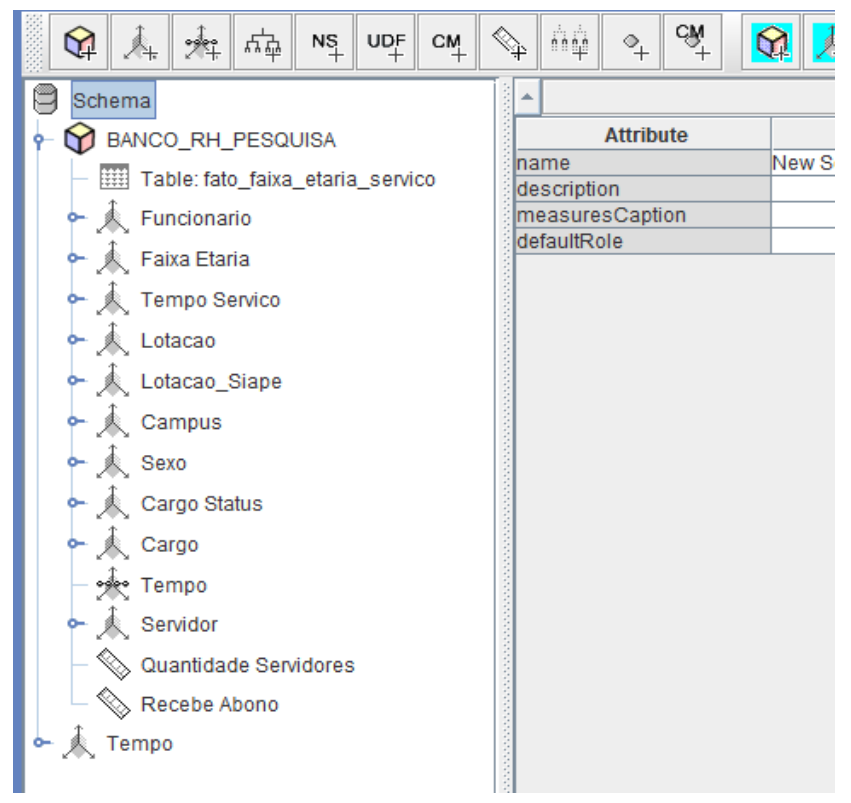

Figura 4 - Cubo OLAP Tabela Fato

Para cada tabela de dimensão (Figura 1) foi criada uma dimensão no cubo, apontando para a dimensão desejada no DW, são elas: Funcionário; Faixa Etária; Tempo Serviço; Lotação; Campus; Sexo; Cargo; Cargo Status; Tempo; e Servidor. A dimensão Tempo foi definida fora do cubo, por ser uma dimensão genérica que pode ser aproveitada em outros cubos no mesmo Schema. As medidas utilizadas nessa pesquisa foram as "Quantidades Servidores" e "Recebe Abono". O próximo passo foi publicar o cubo no sistema Pentaho Server.

Após a criação do Cubo, foi desenvolvido um Job para ordenar as rotinas de transformações do processo ETL para a realizar as cargas nas tabelas STG e DIM. A Figura 5 ilustra o processo ETL para as tabelas DIM realizando a carga no DW na tabela "Fato_Faixa_Etaria_Tempo".

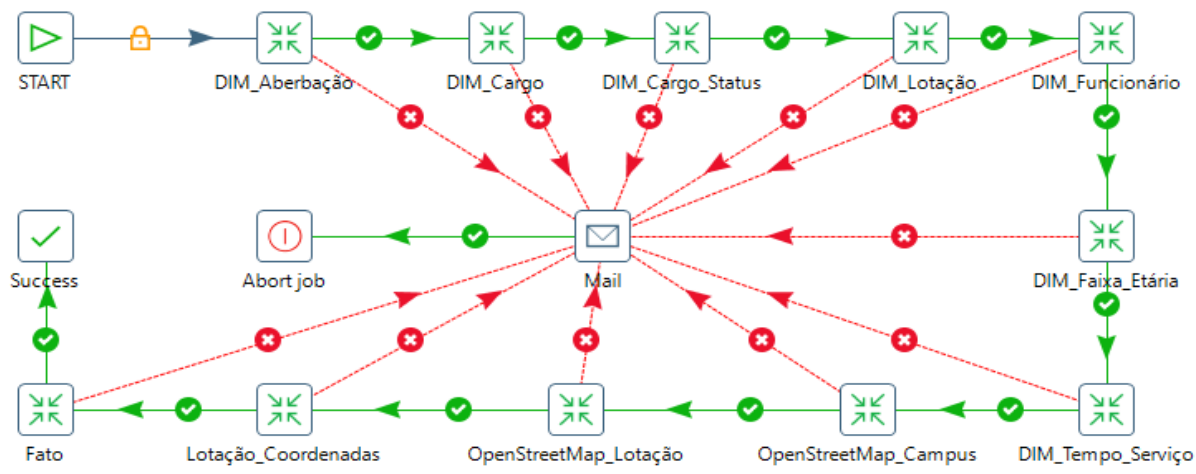

Figura 5: Rotinas job carga Tabela "Fato_Faixa_Etaria_Tempo" 
O Step START (Figura 5) inicia o processo da carga dos dados para o DW, na seguinte ordem: START; DIM_Averbação; DIM_Cargo; DIM_Cargo_Status; DIM_Lotação; DIM_Funcionário; DIM_Faixa_Etária; DIM_Tempo_Serviço; OpenStreetMap_Campus; OpenStreetMap_Lotação; Lotação_Coordenadas; Fato; e Sucess. Caso ocorra algum erro em alguma etapa do processo, um email é disparado para o administrador com log utilizando o Step Mail e a execução é abortada pelo Step Abort $J o b$. A visualização dos resultados pode ser acessada por um navegador Web utilizando a ferramenta CDE Dashboard da Suíte Pentaho.

\section{Resultados}

O desenvolvimento do Data Mart utilizando ferramentas de BI, permite ao gestor extrair informações globais e detalhadas sobre a gestão de pessoas da instituição. Para exemplificar o potencial do DM implementado, a seguir são apresentadas algumas tabelas, gráficos e imagens obtidas com dados relativos à situação real dos servidores da UFV, referentes ao mês de fevereiro de 2021.

A tabela Fato Servidores Faixa Etária e Tempo de Serviço, permitiu analisar dados quantitativos dos servidores ativos por Campus, gênero, faixa etária e tempo de serviço, conforme ilustrado na Figura 6.

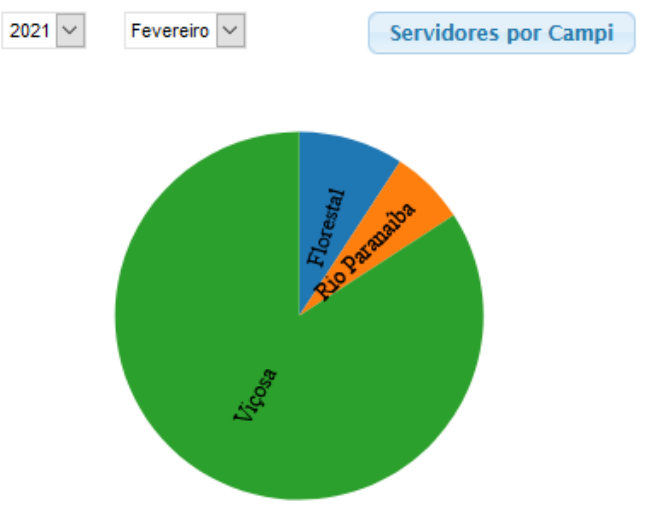

Servidores: Todos os Campi Voltar
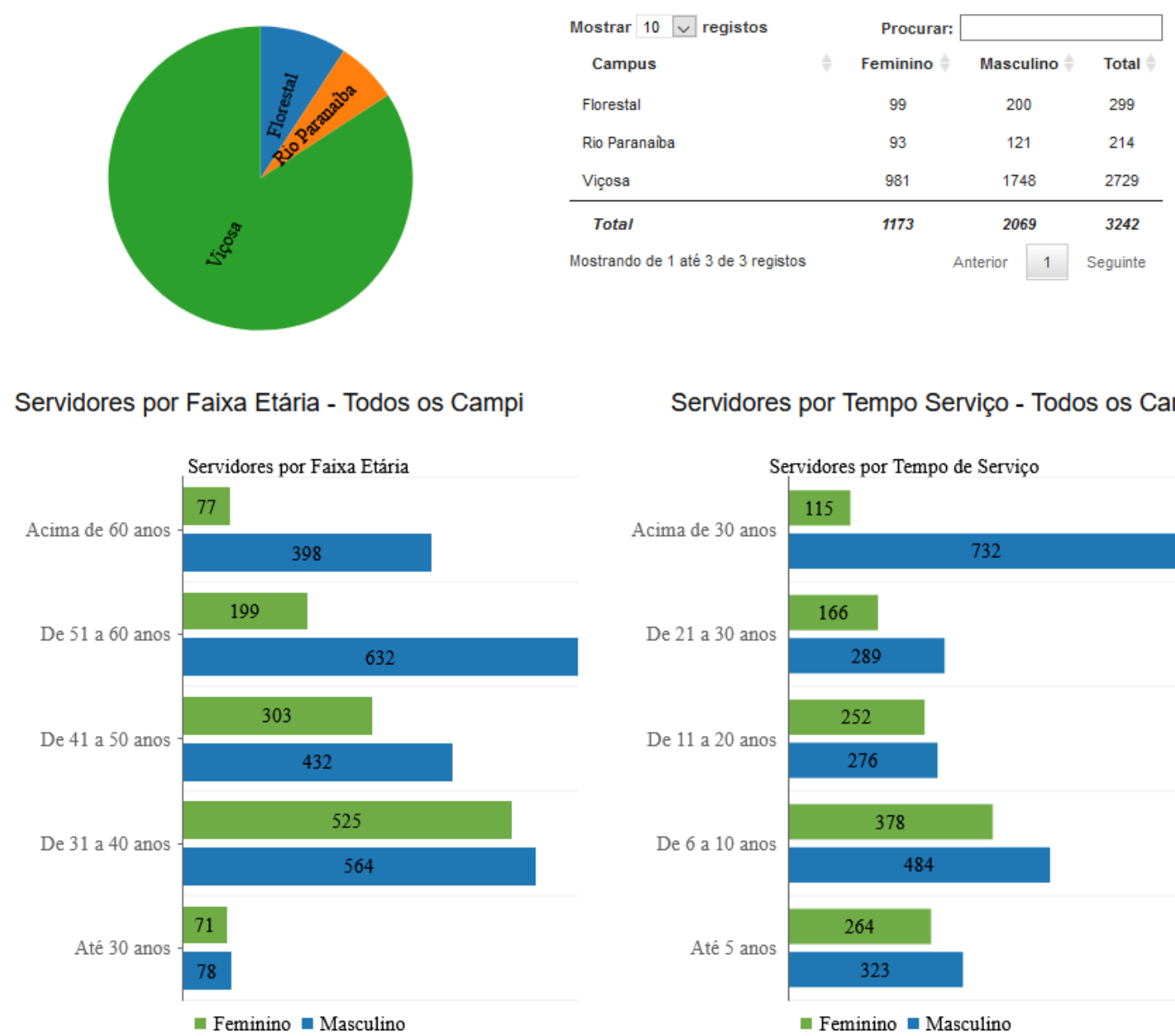

Servidores por Tempo Serviço - Todos os Campi

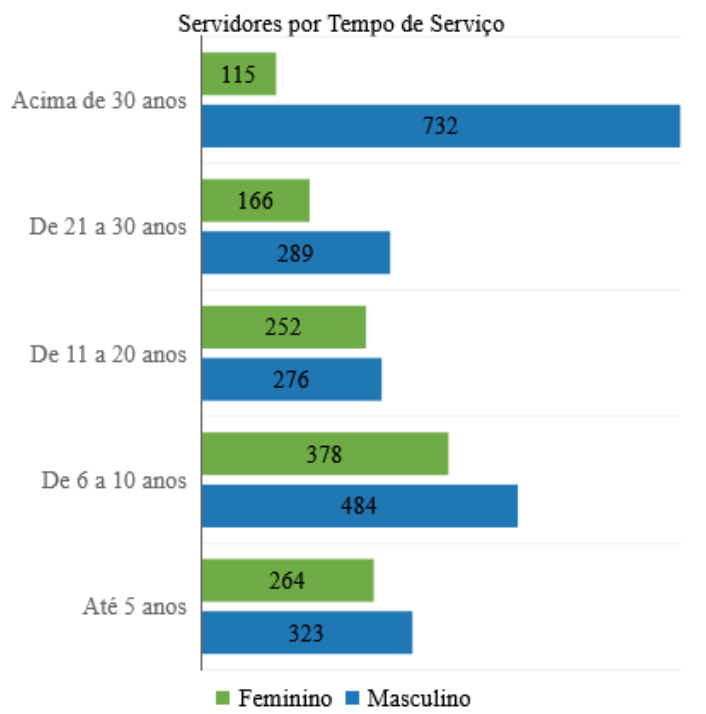

Figura 6: Servidores por Faixa Etária e Tempo de Serviço 
A Figura 6 ilustra o dashboard inicial que, por padrão, apresenta os dados totalizados dos servidores dos três campi da UFV. Ao selecionar um campus no gráfico de pizza, é passado um parâmetro que modifica o dashboard e são apresentados os dados apenas do campus selecionado. Ao clicar no botão "Servidores por Campi" o Dashboard volta a configuração inicial com os dados dos três campi. Observa-se ainda que é possível realizar consultas históricas.

Ao analisar o dashboard da Figura 6, verifica-se um considerável número de servidores classificados na faixa etária com o status "De 51 a 60 anos" e "Acima de 60 anos", resultando em 1.306 servidores, de um total de 3.242 , ou seja, cerca de $40 \%$ do quadro efetivo da Instituição. Já o gráfico de servidores por tempo de serviço apresenta um elevado índice (847) de servidores e servidoras com os status "Acima de 30 anos", apresentando potencial problema a ser enfrentado pelos gestores.

O resultado da geocodificação pode ser visualizado na Figura 7, gerado pela ferramenta do BI com dados do OpenStreetMap. Os três campi são pontuados no mapa, que carrega o quantitativo geral de servidores, divididos em "Docente" e "Técnico ADM". O mapa pode ser apresentado com os dados gerais ou com os dados de cada campus passado por parâmetro no dashboard.

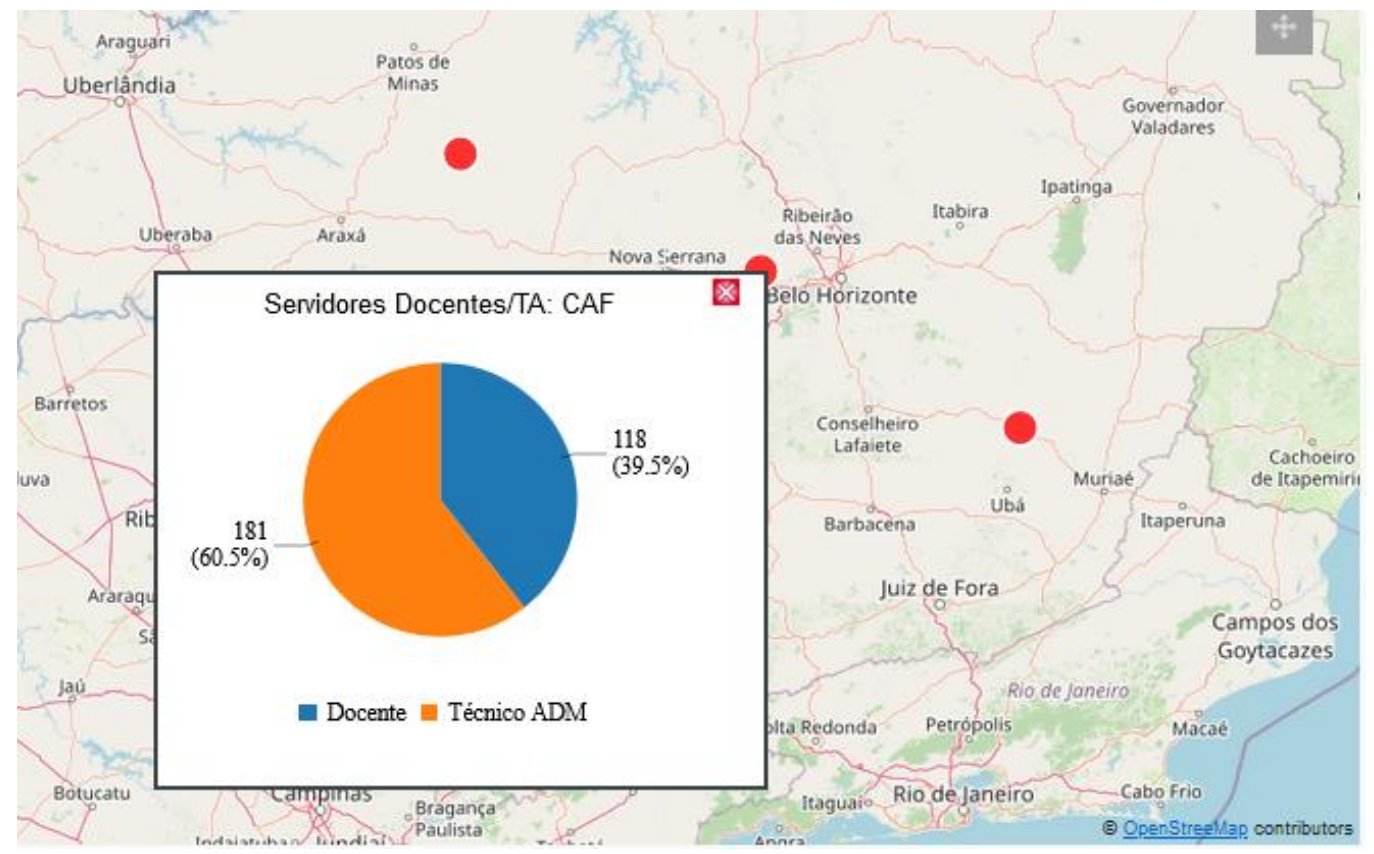

Figura 7: Servidores por Campus por Docente e Técnicos ADM

Outro dashboard aborda o quantitativo dos servidores por cargo, gênero, campi e lotação. A Figura 8 ilustra as lotações por pró-reitorias, centros acadêmicos, campi e o Centro Experimental do Triângulo Mineiro-CTM. Ao clicar em uma barra do gráfico, o layout do dashboard muda para a opção selecionado. Parte da Figura 8 refere-se a uma tabela com os quantitativos dos servidores por lotação e gênero que pode ser mudado de acordo com parâmetro atual do layout. Esse mesmo Dashboard disponibiliza dados quantitativos dos servidores por meio de dois gráficos de barra, com a faixa de etária e tempo de serviço por lotação de acordo com parâmetro atual da tela. 


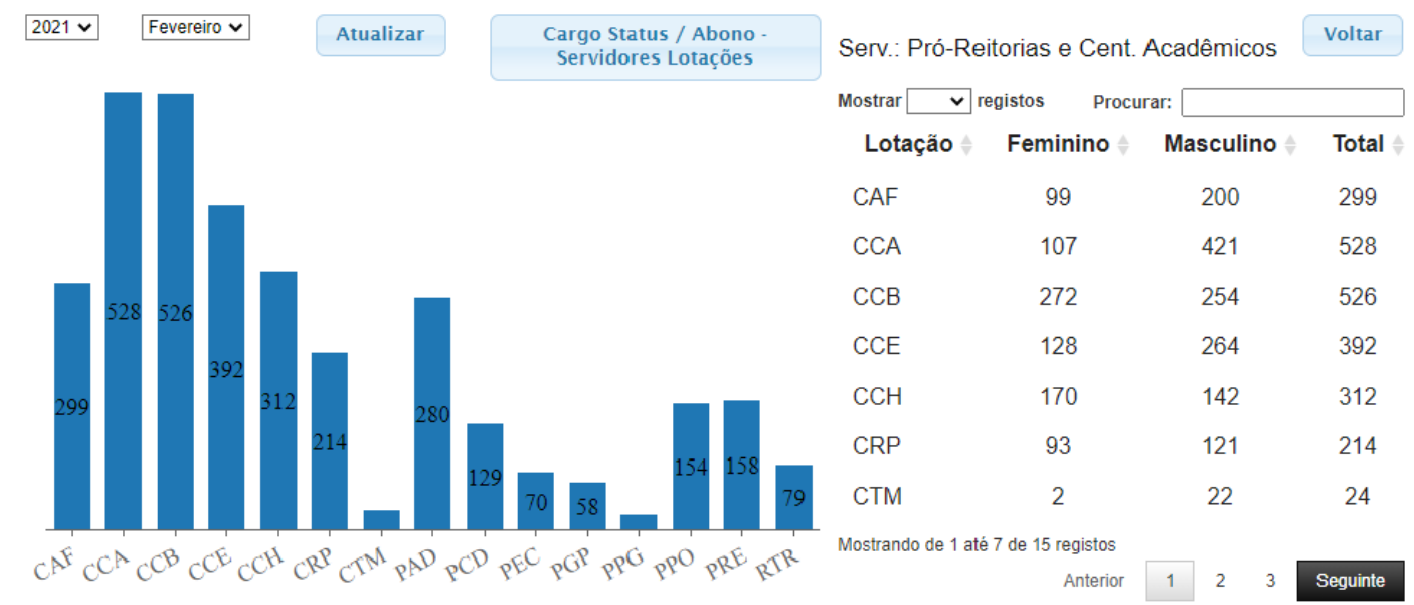

Figura 8: Quantitativos de Servidores por Lotação

Esse dashboard apresenta ainda o quantitativo dos servidores da Figura 8, que pode ser visualizado com a geolocalização das lotações, Figura 9. Ao clicar em uma barra do gráfico da Figura 8, é passado um parâmetro para o mapa que atualiza os dados, de acordo a lotação desejada.

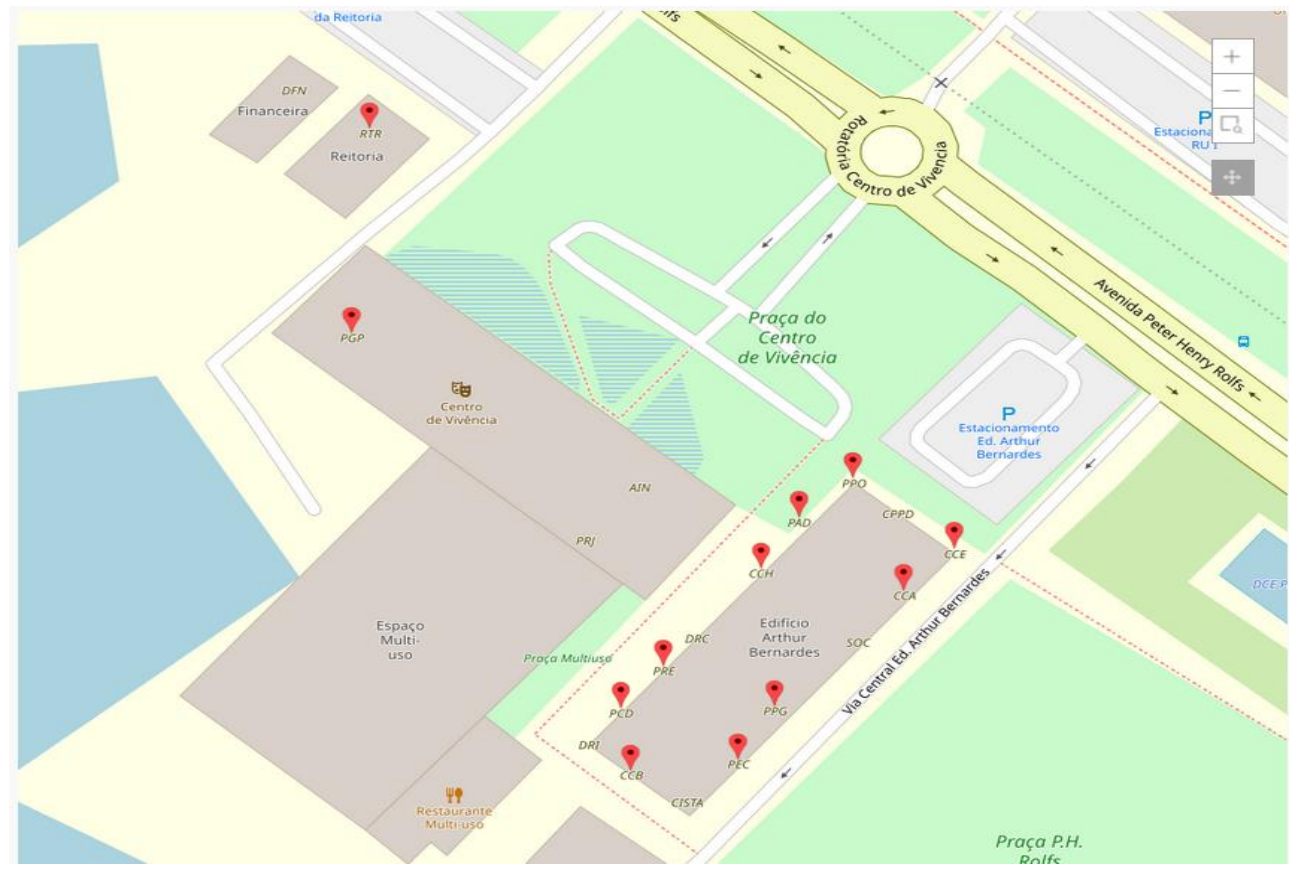

Figura 9: Geolocalização das Lotações

Quando acionado, o botão 'Cargo Status / Abono - Servidores Lotações" da Figura 8, é passado um paramento da lotação atual do dashboard para um novo dashboard. Neste novo painel são disponibilizadas as informações sobre o quantitativo de servidores por cargo, que recebem o "Abono Permanência", e podem solicitar a aposentadoria a qualquer momento (Figura 10), além de informações do status do cargo: Ativo - pode realizar concurso para cargos vagos; Vedado - Concursos para esses cargos estão vedados; Extinto - não pode realizar concursos para preenchimento dos cargos vagos.

O gráfico de barra da Figura 10, mostra apenas o quantitativo de servidores que recebem o "Abono Permanência", por status do cargo. Além disso o mesmo gráfico informa que o número desses servidores com o status de "Extinto" e "Vedado" 
corresponde a mais de 50\% do total de 546 servidores. A Figura 10 ilustra também um quadro geral de todos os servidores, incluídos os que recebem o "Abono Permanência", por gênero e status do cargo. Pode-se observar um número elevado de servidores com o status cargo "Extinto" e "Vedado", resultando em cerca de $32 \%$ do quadro. Fica uma incógnita para os gestores para saber como esses servidores poderão ser substituídos, sem a realização de concursos e recursos.

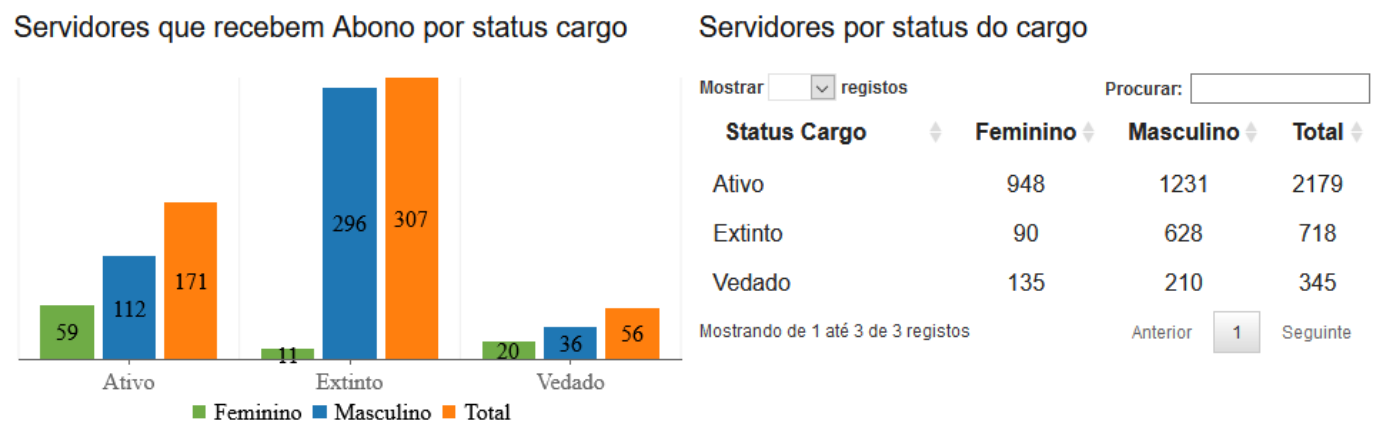

Figura 10: Quantitativos de servidores que recebem Abono de Permanência por Status do Cargo e quantitativo de servidores por status do cargo

O mesmo dashboard disponibiliza uma tabela com as informações dos cargos por lotações com os seguintes campos: Unidade; Sigla; Nome; Cargos (Ativo, Vedado Concurso e Extinto); Carreiras (Docente e Técnico Administrativo) com o quantitativo Total e o quantitativo dos que recebem Abono de Permanência; e o Total Geral de servidores. Ao acionar a coluna "Unidade" da tabela o usuário é direcionado para um novo dashboard com as informações somente daquele órgão, proporcionando uma consulta mais detalhada.

\section{Conclusões}

O Data Mart desenvolvido com as ferramentas do BI e com a integração de outros sistemas possibilitou a obtenção de informações dos servidores por campi, faixa etária, tempo de serviço, localização geográfica por lotação e cargo.

A pesquisa permitiu analisar o quadro de força de trabalho dos servidores na UFV, apontando para o elevado número de servidores que podem desligar-se da Instituição a qualquer momento, além de evidenciar a impossibilidade de reposição de vagas de cargos extintos ou vedados para concurso.

Todo o processo foi realizado utilizando apenas sistemas de software livre, o que implica em disponibilizar uma ferramenta de BI para tomada de decisão gerencial sem adicionar custos com aquisição de software proprietário. Acredita-se que esta ferramenta possa ser implementada em outras IFEs com pouco esforço, uma vez que além do banco de dados do SIAPE, o DM extrai dados de um banco de dados próprio da UFV.

Os dois indicadores proposto pela pesquisa foram implementados na PGP e como trabalhos futuros tem-se a possibilidade de realizar estudos sobre a projeção de aposentadorias por cargo e o seu impacto na reposição do quadro de servidores.

\section{Agradecimentos}

Os autores agradecem à Universidade Federal de Viçosa pela autorização de uso de dados reais. Este projeto foi parcialmente financiado com recursos da Fapemig - Fundação de Amparo à Pesquisa do Estado de Minas Gerais. 


\section{Referências}

Brasil. Decreto-Lei $\quad \mathrm{N}^{\mathrm{o}}$ 6.096. (2007). Disponível em: http://www.planalto.gov.br/ccivil_03/_ato2007-2010/2007/decreto/d6096.htm. Acesso em: 06 jun. 2020.

Decreto-Lei

http://www.planalto.gov.br/ccivil_03/_ato2007-2010/2010/decreto/d7232.htm. Acesso em: 06 jun. 2020.

. Decreto-Lei $\quad \mathrm{N}^{\mathrm{o}}$

7.485/2011.

(2011).

Disponível

em: http://www.planalto.gov.br/ccivil_03/_ato2011-2014/2011/decreto/d7485.htm. Acesso em: 16 ago. 2020.

GeoProfile. (2020). "Perfil UML Geoprofile para Banco de Dados Geográfico". Disponível em: <http://www.dpi.ufv.br/projetos/geoprofile/download.html>. Acessado em 15 junho 2020.

Inmon, W. B. (1997). Como construir o Data Warehouse. Rio de Janeiro: Campus.

Kimball, R. (1998). Data Warehouse Toolkit: Técnicas para Construção de Data Warehouse Dimensionais. São Paulo: Makron Books.

Lisboa-Filho, J., Sampaio, G. B., Nalon, F. R., and Borges, K. A. D. V. (2010)". A UML Profile for Conceptual Modeling in GIS Domain", CAiSE 2010 Workshop, Hammamet, Tunisia.

Machado, F. (2007) Tecnologia e Projeto de Data Warehouse: Uma Visão Multidimensional. São Paulo: Érica.

Medina, Fernando, Francisco Fariña, and Wilson Castillo-Rojas. (2018). "Data Mart para obtención de indicadores de productividad académica en una universidad." Ingeniare. Revista chilena de ingeniería 26 (2018): 88-101.

Nominatim. (2020) "Nomination com Dados do OpenStreetMap". Disponível em: https://operations.osmfoundation.org/policies/nominatim/>. Acessado em 20 maio 2020.

OpenStreetMap. (2020). "Mapeamento Colaborativo". Disponível em: https://www.openstreetmap.org. Acessado em 20 de maio 2020.

Primak, F. V. (2008). Decisões com BI (Business Intelligence). Rio de Janeiro: Ciência Moderna.

Rob, P. and Coronel, C. (2010). Sistemas de Banco de Dados: Projeto, Implementação e Administração. São Paulo: Cengage do Brasil.

Suíte Pentaho. (2020). Disponível em: https://sourceforge.net/projects/pentaho/. Acessado em 10 maio de 2020.

Takashina, N. and Flores, M. (1996). Indicadores da qualidade e do desempenho: como estabelecer metas e medir resultados. Rio de Janeiro: Qualitymark. 